\title{
Virtual Machines Migration based on Greedy Algorithm in Cloud Computing
}

\author{
Somayeh Soltan Baghshahi \\ Department of Computer \\ Engineering, Tehran North \\ Branch, Islamic Azad University, \\ Tehran, Iran
}

\author{
Sam Jabbehdari \\ Department of Computer \\ Engineering, Tehran North \\ Branch, Islamic Azad University, \\ Tehran, Iran
}

\author{
Sahar Adabi \\ Department of Computer \\ Engineering, Tehran North \\ Branch, Islamic Azad University, \\ Tehran, Iran
}

\begin{abstract}
Cloud computing is a term that used for distributed computing, on a network. It is meant network-based services that these services are provided through virtual ISPs. Most of the old virtual machine migration techniques concentrated on the migration of a single virtual machine. These techniques are insufficient, when the whole virtual cluster needs to be migrated. In these methods, data replication in each round leads to many computational overheads.

In this paper, a method is proposed for virtual machine migration from one data center to another. The proposed method uses the greedy algorithm for virtual machine selection. Virtual machines within a cluster are given priority, which reduces the total migration time of a cluster. This method is simulated in CloudSim tools in Eclipse. Simulation results show that the bandwidth parameter has the important role in the virtual machine migration.
\end{abstract}

\section{General Terms}

Distributed Systems, Virtualization, Greedy Algorithm

\section{Keywords}

Cloud Computing, Virtual Machine, Migration.

\section{INTRODUCTION}

When computers come to human life, it passes many years. Over the years, tend to development has led to the development of new technologies. Furthermore, when the Internet is completely useable for the user, it passes many years. The Internet has created a dramatic change in information exchange. Of course, at that time, someone did not think that from the Internet can be used as a powerful processing system. Among the emerging technologies, cloud computing has taken a step beyond other technologies.

Cloud computing is a technology of data processing where computer resources and capacity are provided to the user as an Internet service. The user has access to own data, but should not care about the infrastructure, operating system, and the software. Cloud computing is a paradigm in which information is permanently stored in servers on the Internet and cached temporarily on clients who include desktop computers, entertainment centers, table computers, notebooks, wall computers, handheld devices, sensors, and monitor [1]. The founder of the cloud computing concept was John McCarthy, who suggested in the 1960s that the future calculations would be implemented through public utilities. The ideology of cloud computing gained popularity in 2007, thanks to the rapid evolution of communication channels and an increase in the geometric progression of the needs of both business and private users to expand their information systems [1] .

Cloud computing has recently attracted significant attention from both industry and academia for its ability to deliver IT services at a lower barrier to entry in terms of cost, risk, and expertise, with higher flexibility and better scaling ondemand. While many cloud users' early successes have been realized using a single cloud provider, using multiple clouds to deliver services and having the flexibility to move freely among different providers is an emerging requirement [2].

The best mechanism for implementation of cloud is Virtualization technology. Virtualization is an old technology, which was used for the computer systems in 1964. Virtualization is meant to make a copy of everything, including the operating system, storage space and network equipment and so on.

Virtualization technology has several types, each of which will be utilized for special applications. A unique feature of Virtualization is a virtual machine live migration technique. So that, when a virtual machine is running and providing service to users, with different reasons, such as detect a failure in the physical machine, the system automatically decides to virtual machine migration from the physical machine to another, without occurs an interruption in service.

Therefore, virtual machine migration will be done with low latency. In cloud environments, services are provided through virtual machines.

\section{RELATED WORK}

Cloud computing provides a way to maximize the capacity and capabilities without investing in infrastructure. The principal purpose of applying the technique migration, load balancing, fault tolerance, energy management and maintenance of servers. Its principal function is to improve the service. For the implementation of this technique has been proposed so many methods.

Pre-copy migration tries to tackle problems associated with earlier designs by combining a bounded iterative push step with a final and typically very short stop-and-copy phase. The core idea of this design is that of iterative convergence. It involves iterating through multiple rounds of copying in which the virtual machine memory pages that have been modified during the previous copy are resent to the destination on the assumption that at some point the number of modified pages will be small enough to halt the virtual machine temporarily, copy the (small number of) remaining pages across, and restart it on the destination host. The result of this research is reducing Down Time [3].

Agarwal et al [4] studied the advantages and disadvantages of virtual machine migration and among existing methods; they 
evaluated the Pre-Copy technique and proposed a framework for virtual machines migration based on this approach.

In the post-copy technique, first suspends the migrating virtual machine at the source node, copies minimal processor state to the target node, resumes the virtual machine, and begins fetching memory pages over the network from the source. The manner in which pages are fetched gives rise to different variants of post-copy, each of which provides incremental improvements. The result of this research is to reduce the number of pages transmitted and total migration time compared to the Pre-Copy technique [5].

Most of the previous live virtual machine migration techniques concentrated on the migration of a single virtual machine which means these techniques are insufficient when the multiple virtual machine need to be migrated. Therefore, Ye et al [6] proposed a framework for migrating Virtual clusters. They evaluated the performance and overhead of virtual clusters live migration.

\section{VIRTUALIZATION AND VIRTUAL MACHINE MIGRATION}

Virtualization is the key technology that enables the emerging cloud computing paradigm, because it allows resources to be allocated to different applications on-demand and hides the complexity of resource sharing from cloud users. Virtual machines are generally employed in different types of cloud systems as containers for hosting application execution environments and provisioning resources. For example, in Infrastructure-as-a-Service (IaaS) clouds [7], virtual machines are directly exposed to users to deliver a full computer infrastructure over the Internet; In Platform-as-a-Service (PaaS) clouds, also, virtual machines are used by the clouds internally to manage resources across the application execution platforms delivered to users.

Virtual machine migration is a unique capability of system virtualization which allows an application to be transparently moved from one physical host to another and to continue its execution after migration without any loss of progress. It is generally done by transferring the application along with its VM's entire system state, including the state in CPU, memory, and sometimes disk too, from the source host to the destination host. VM migration is an important means for managing applications and resources in large scale virtualized data centers and cloud systems. It enables resource usage to be dynamically balanced in the entire virtualized system across physical host boundaries, and it also allows applications to be dynamically relocated to hosts that can provide faster or more reliable executions [8].

Virtual machine migration takes a running virtual machine and moves it from one physical machine to another. This migration must be transparent to the guest operating system, applications running on the operating system, and remote clients of the virtual machine. It should appear to all parties involved that the virtual machine did not change its location. The only perceived change should be a brief slow down during the migration and a possible improvement in performance after the migration because the virtual machine was moved to a machine with more available resources [7]. Live virtual machine migration technologies have proven to be a very effective tool to enable data center management in a non-disruptive way. Virtual machine migration techniques include pre-copy, post-copy, Three-Phase Migration, CR/TR Motion, Heterogeneous and Dependency-Aware.

These techniques are not efficient for migration a cluster of virtual machines.
Two methods are proposed for migration a cluster of virtual machines, Concurrent migration and sequential migration [6].

\section{- Concurrent Migration}

In this method, a cluster of virtual machines concurrently sent from a source to a destination. This method is used when the size of the virtual machines within a cluster is small.

\section{- Sequential Migration}

In this method, the virtual machines are placed in a queue and they are sent consecutively. They are used for mutual migration.

These methods are shown in Figure 1.
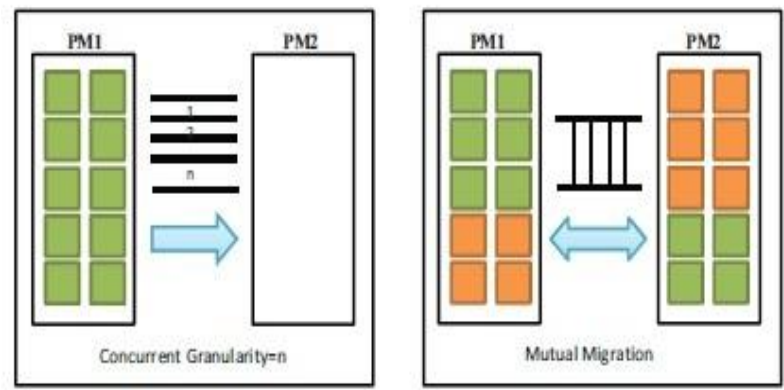

Fig 1: Concurrent and sequential migration [6]

In the concurrent migration of virtual machines [6] is required much bandwidth to transfer data. Therefore, sequential migration has a better performance than the concurrent migration.

Average migration time is depending on the memory size. If memory size of virtual machines within a cluster is large, the more data is transmitted over the network. Therefore, the migration time is equal to the sum of the migration time of virtual machines within a cluster, which increase linearly.

In the proposed method, we use the greedy algorithm and the sequential migration method, so that the changes will lead to changes in migration time.

\section{GREEDY ALGORITHM}

In the greedy algorithm, data items in sequence, each time taking the one that is deemed "best" according to some criterion, without regard for the choices it has made before or will in the future. A greedy algorithm arrives at a solution by making a sequence of choices, each of which simply looks the best at the moment.

A greedy algorithm starts with an empty set and adds items to the set in sequence until the set represents a solution to an instance of a problem.

There are following components in per iteration:

- A selection procedure chooses the next item to add to the set. The selection is performed according to a greedy criterion that satisfies some locally optimal consideration at the time.

- A feasibility check determines if the new set is feasible by checking whether it is possible to complete this set in such a way as to give a solution to the instance.

- A solution check determines whether the new set constitutes a solution to the instance [9].

\subsection{Proposed Method}

In this method, the selection procedure for virtual machine migration is the memory size of the virtual machines and storage space of the destination physical machine. So that, at 
each step, a comparison is done between the memory size of virtual machine and storage space of the physical machine, if the memory size of virtual machine was smaller than storage space of the physical machine, it will be selected for migration.

\section{Proposed Algorithm for Virtual Machines Migration}

1. input:

2. Hostlist, Vmlist //Sorted Desc

3. Curent_Time

4. Link_Speed

5. VmMigration_Time

6. VmMigrationList_Time

7.

8. For $i: 0$ to Hostlist

9. host: Host_LargSize in Hostlist

10. while host $>0$

11. $v m$ : VM_LargSize in Vmlist

12. for $j: 1$ to Vmlist

13. If $v m>$ host then

14. $v m: v m++$ in vmlist

$15 . \quad$ else

16. host:host - vm (size)

17. $v m$ is in Migration

18.

VmMigrationList_Time:Curent_Time +

19. ( $v m /$ Link_Speed)

20.

$v m: v m++$ in Vmlist

21. host:host++ in Hostlist

Fig 2: Pseudo-code of the greedy algorithm for virtual machines migration

\subsection{The proposed algorithm for Migration}

In this algorithm, has been considered two lists for a cluster of virtual machines and destination physical machines. These two lists are sorted by descending order.

The steps of the proposed algorithm for virtual machines migration is as follows:

- The Select an item from the list of destination physical machines.

- Until the selected element (physical machine) has enough storage space, perform the following steps:

Step 1: Select an item from the list of virtual machines.

Step 2: Comparing the size of the selected virtual machine with storage space of the physical machine.

If the size of virtual machine was greater, go to step 1 and select the next virtual machine. Otherwise, the virtual machine is migrated to the selected physical machine.

The pseudo-code of the proposed algorithm is shown in Figure 2.

\section{SIMULATION}

To simulate the proposed algorithm, we define a scenario for virtual machine migration. In this scenario, two data center are considered that there are five physical machines in each data center. The first data center is contained ten virtual machines and the second data center is contained five virtual machines. To implementation of the proposed algorithm, we used CloudSim Tool $[10,11]$ in Eclipse. One of the features of this tool is the implementation of virtual machine migration. The simulation results are shown below. In our proposed method, we used of the greedy algorithm and the sequential technique, so that, with the change of memory size, the total migration time is changed. These changes are shown in Figure3.

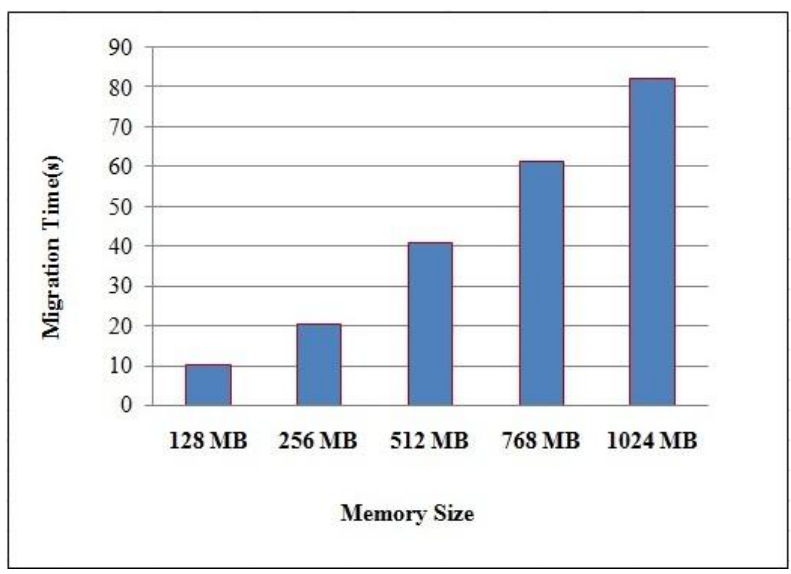

Fig 3: The Total Migration Time in Proposed Method

Also, with the change of the number of virtual machines in the cluster, the total migration time is changed. These changes are shown in Figure 4.

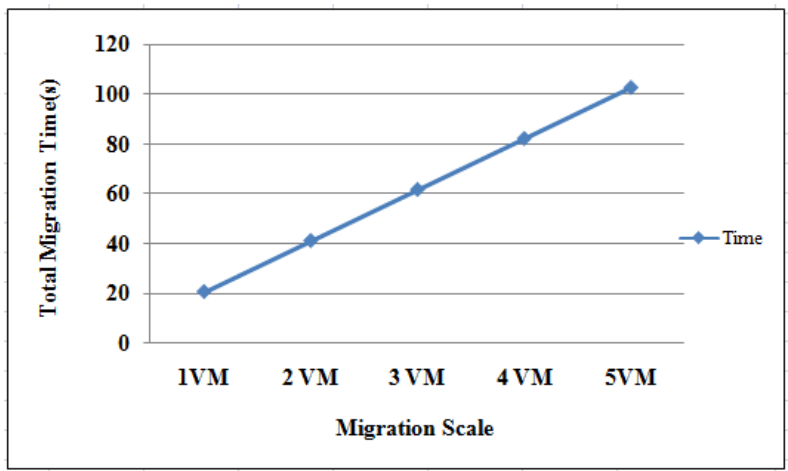

Fig 4: The Total Migration Time for cluster with different numbers of $\mathrm{VM}$

The comparison of changes in migration time for a cluster with virtual machines with different sizes, in serial method and our proposed method are shown in Figure 5. 


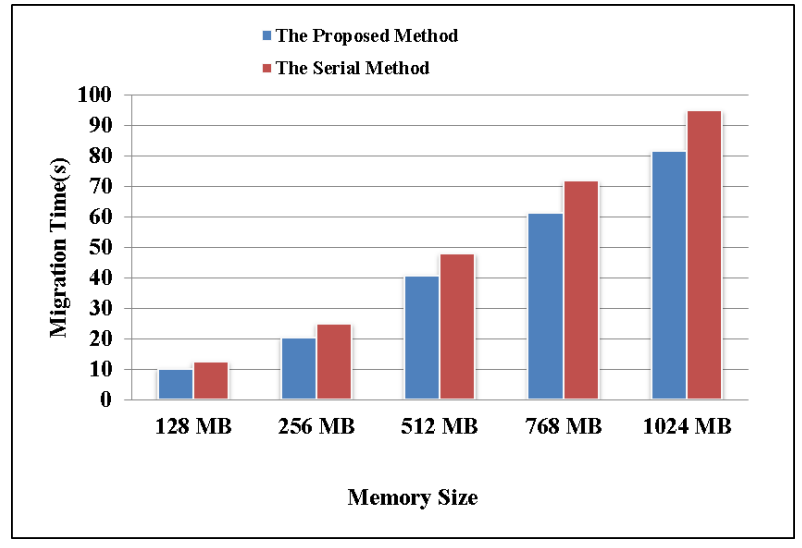

Fig 5: Comparison of Migration Time for clusters in two methods

Also, the comparison of changes total migration time of virtual clusters with different numbers of virtual machines in serial method and our proposed method are shown in Figure6.

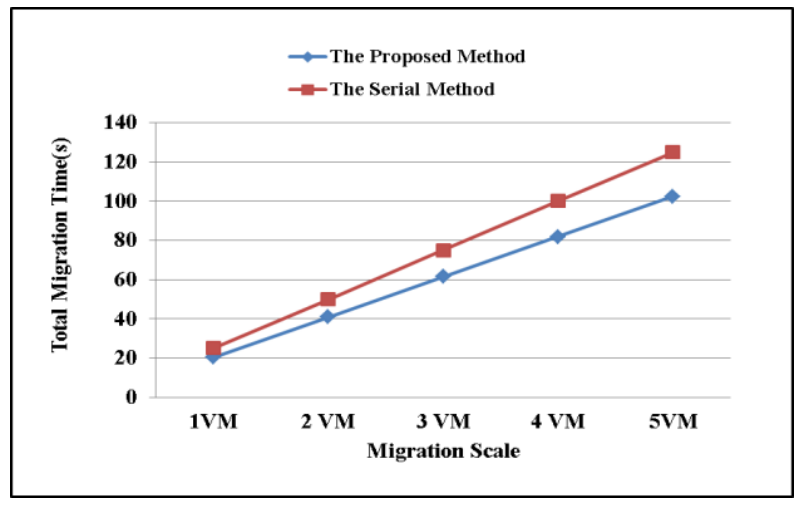

Fig 6: Comparison of Total Migration Time for cluster with different numbers of VM in two methods

\section{CONCLUSION}

According to studies, there are many challenges and weaknesses in providing service to customers through the Internet, Such as the lack of service quality due to lack of sufficient computational resources, geographical distance between clients and service providers, insufficient bandwidth and so on. Therefore, cloud computing using virtualization technology can improve these weaknesses. Most of the previous virtual machine migration techniques concentrated on the migration of a single virtual machine which means these techniques are insufficient when the whole virtual cluster need to be migrated. In these methods, data replication in each round leads to many computational overheads. Therefore, in this paper we proposed a method the based on the greedy algorithm that the set of virtual machines can be moved to another location. In our method, the priority is considered for the virtual machines lead to reduce of the total migration time.

\section{REFERENCES}

[1] V.V. Arutyunov, "Cloud Computing: It's History of Development, Modern State, and Future Considerations", Scientific and Technical Information Processing, Vol. 39 , No. 3, pp. 173-178, 2012.

[2] J. Zeng, T. S. Eugene, K. Sripanidkulchai, "WorkloadAware Live Storage Migration for Clouds", Newport Beach, California, USA, VEE'11, March 9-11, 2011.

[3] C. Clark, K. Fraser, S. Hand, J. Hansen, "Live Migration of Virtual Machine", NSDI'05 Proceedings of the 2nd conference on Symposium on Networked Systems Design \& Implementation - Volume 2, pp.273 - 286, 2005.

[4] A. Agarwal, S. Raina, "Live Migration of Virtual Machines in Cloud", International Journal of Scientific and Research Publications, Volume 2, Issue 6, June 2012.

[5] M.R.Hines, U.Eshpande, K.Gopalan, "Post-Copy Live Migration of Virtualization of Virtual Machine" International Conference on Virtual Execution Environment (VEE), pp. 14-26, March 2009.

[6] K. Ye, x. Jiang, R.Ma, F.Yan, "VC-Migration: Live Migration of Virtual Clusters in the Cloud", ACM/IEEE 13th International Conference on Grid Computing, PP. 209-218, 2012.

[7] M. Nelson, B. Lim, and G. Hutchins, "Fast transparent migration for virtual machines", in Proceedings of the annual conference on USENIX Annual Technical Conference, p. 25. 2005.

[8] Y.WU, M.Zaho, "Performance Modeling of Virtual Machine Live Migration", IEEE International Conference on Cloud Computing (CLOUD), 2011.

[9] R. Neapolitan, K. Naimipour, "Foundations of Algorithms Using C++ Pseudocode", Jones and Bartlett Publishers, chapter 4, 2004.

[10] R. Calheiros, R. Ranjan, A. Beloglazov, F. De Rose and R. Buyya, "CloudSim: A Toolkit for Modeling and Simulation of Cloud Computing Environments and Evaluation of Resource Provisioning Algorithms", Software: Practice And Experience Vol.41, no.1, PP. 2350, 2011.

[11] R. Buyya, R. Ranjan, and R.N. Calheiros, "Modeling and simulation of scalable cloud computing environments and the cloudsim toolkit: Challenges and opportunities", Proc. Of the 7th High Performance Computing \& Simulation Conference (HPCS'09), IEEE Computer Society, 2009. 